\title{
ASCA Observations of Supernova Remnants
}

\author{
By HIROSHI TSUNEMI AND THE ASCA TEAM
}

\author{
Department of Earth and Space Science, Faculty of Science, Osaka University, Japan
}

\begin{abstract}
We present here preliminary results of the $A S C A$ satellite. $A S C A$ is equipped with $\mathrm{X}$-ray telescopes that can observe the energy range up to $12 \mathrm{keV}$. There are two types of detector systems: GIS and SIS. The energy resolution of the SIS is $130 \mathrm{eV}$ (FWHM at $6 \mathrm{keV}$ ) and can resolve emission lines clearly. For the PV phase, we planned to observe about 150 sources. Among them, there are 23 SNR's, some of which are presented here. We will be able to study the evolution of thin hot plasma in the SNRs.
\end{abstract}

\section{Introduction}

The fourth Japanese X-ray Astronomy satellite was successfully launched on February 20, 1993, from Kagoshima Space Center. The satellite's pre-launch name, Astro-D, was changed to it ASCA once it achieved orbit. $A S C A$ is equipped with four thin foil X-ray mirror telescopes (XRT) that can collect X-rays up to $12 \mathrm{keV}$. Fig. 1 shows the effective area of the XRT. The XRT has a point spread function (PSF) with a half power diameter (HPD) about 2.7 arcmin. There is a sharp core of about 20 arcsec diameter in the PSF that enables us to separate point sources separated by less than one arcmin.

$A S C A$ has two types of detectors: one is the imaging gas scintillation proportional counter, (IGSPC, Ohashi et al, 1991) and the other is the X-ray CCD camera (Burke, et al., 1993). They are called the gas imaging spectrometers (GIS) and the solid-state imaging spectrometers (SIS), respectively. There are two GIS detectors and two SIS detectors. Each detector is at the focal plane of its own XRT. One SIS detector has 4 CCD chips which can observe a field of view of $22 \times 22 \operatorname{arcmin}^{2}$. The basic parameters for the GIS and SIS are summarized in table 1.

The GIS can observe the whole effective field of view of the XRT and has a time resolution down to $61 \mu \mathrm{sec}$. Although it cannot observe the energy range below $0.7 \mathrm{keV}$ due to the thickness of its beryllium window, it can observe the energy range up to 12 $\mathrm{keV}$ with high detection efficiency (greater than $70 \%$ ). The SIS has a smaller field of view, poorer time resolution, but a broader energy range and higher energy resolution. The CCD chip is designed for X-ray studies in orbit: low read-out noise, thick depletion region and radiation-hardness (Burke, et al., 1991). The detection efficiency around 10 $\mathrm{keV}$ is inferior to that of the GIS. Taken together, these two detector systems complement each other.

The satellite pointing has a sun angle constraint. The optical axis of the XRT is the $\mathrm{Z}$-axis while the solar paddle is facing the $\mathrm{Y}$-axis. We have to point the satellite such that the sun should be placed within 30 degree from the Y-axis. Given this constraint, we can access any part of the sky in six months.

At the end of March, 1993, all the detector systems were activated for initial checkout. All the instruments functioned properly. In mid-April, performance of verification (PV) phase started. It would last about 6 months. Then, the guest observer (GO) phase would start. 
TABLE 1. The basic properties of GIS and SIS.

\begin{tabular}{lll}
\hline \hline Item & GIS & SIS \\
\hline field of view (arcmin) & $40 \phi$ & $22 \times 22$ \\
energy range (keV) & $0.7 \sim 12$ & $0.4 \sim 12$ \\
energy resolution (keV) & 0.45 & 0.13 \\
(FWHM at 6 keV) & & \\
\hline
\end{tabular}

TABle 2. The SNRs to be observed during the PV phase

\begin{tabular}{llll}
\hline \hline Crab & Cassiopeia-A & PSR0540-69 & Kes 75 \\
Puppis A & Kepler & SN1006 & Kes73 \\
W49B & W50 & RCW 86 & RX04591+5147 \\
Vela-X & CTB80 & SN1987A & MSH 15-52 \\
E0102-72 & G292.0+1.8 & Tycho-SNR & N132D \\
Cygnus Loop & G21.5-0.9 & RCW 103 & \\
\hline \hline
\end{tabular}

\section{Observation plan during the PV phase}

During the PV phase, $A S C A$ will observe about 150 targets. There are seven categories: AGNs, Binaries, Clusters, CXB (Cosmic X-ray Background), Galaxies, Stars and SNRs. In the SNR category, there are 23 targets listed in table 2.

\section{Observation results}

We present here the X-ray images and spectra for various SNR observed during the $\mathrm{PV}$ phase. Most of them show interesting spectral features rather than the temporal variation. Therefore, the focus of this paper will be on the results obtained with the SIS. Most of the background generated by the particles can be easily separated from the X-ray events by the difference in charge distribution in the CCD. The X-ray background level of the SIS (several $\times 10^{-4}$ counts $\mathrm{s}^{-1} \mathrm{~cm}^{-2} \mathrm{keV}^{-1}$ ) is so low that we need not subtract the background spectrum, particularly for a point-like source. Therefore, all the spectra shown in this paper have not been background subtracted. Extended sources like the Cygnus Loop are slightly affected by the diffuse X-ray background. Since the in-orbit self calibration for the detectors has not been finished by the writing of this paper, all the data shown here are preliminary.

The energy resolving power of the SIS can clearly separate the K- $\alpha$ emission lines from various ion species. Typical $\mathrm{K}-\alpha$ lines resolved with the SIS are listed in table 3 . Among others in the energy range between $0.4 \mathrm{keV}$ and $12 \mathrm{keV}$, there are $F e-L$ emission line blends distributed from $0.7 \mathrm{keV}$ to $1.2 \mathrm{keV}$.

\subsection{The Crab Nebula}

We observed the Crab Nebula at various locations in the field of view to study the image quality of the XRT. The XRT functions properly as we expected based on the ground 


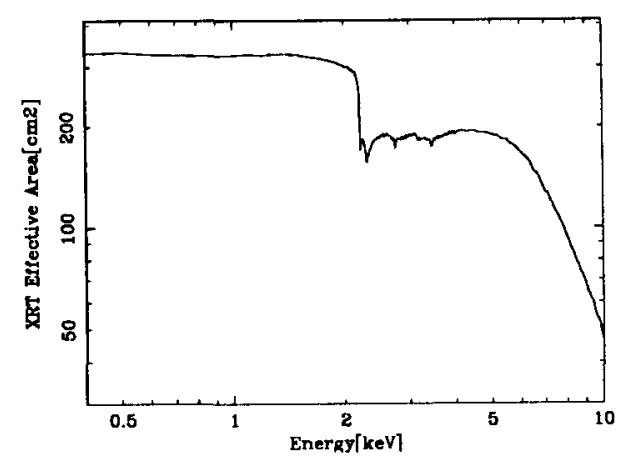

FIGURE 1. The effective area of one XRT.

TABLE 3. $\mathrm{K} \alpha$ emission line energies from various ions

\begin{tabular}{rrrrrrrr}
\hline \hline ion & $\mathrm{keV}$ & ion & $\mathrm{keV}$ & ion & $\mathrm{keV}$ & ion & $\mathrm{keV}$ \\
\hline O VII & $\mathbf{0 . 5 7}$ & $\mathrm{Mg}$ XI & 1.35 & S XV & 2.46 & Ca XIX & 3.91 \\
O VIII & $\mathbf{0 . 6 5}$ & $\mathrm{Mg}$ XII & 1.47 & S XVI & 2.62 & Ca XX & 4.11 \\
Ne IX & $\mathbf{0 . 9 2}$ & Si XIII & 1.86 & A XVII & 3.14 & Fe XXV & 6.70 \\
Ne X & $\mathbf{1 . 0 2}$ & Si XIV & 2.01 & A XVIII & 3.31 & Fe XXVI & 6.96 \\
\hline \hline
\end{tabular}



Figure 2. The X-ray spectrum of the Crab nebula.

calibration. Fig. 2 shows the X-ray spectrum of the Crab nebula obtained with the SIS. It can be well fitted with a power law spectrum without emission lines. There are several spectral features due to the detector system. The clear features seen here are due to the $A u-M$ edges $(2.2 \sim 3.4 \mathrm{keV})$, the $S i-K$ edge $(1.8 \mathrm{keV})$, the $A l-K$ edge $(1.5 \mathrm{keV})$, the $O-K$ edge $(0.53 \mathrm{keV})$. The $A u-M$ edges come from the gold coating on the XRT surface. The $A l-K$ edge comes from the optical block filter placed just above the CCD 


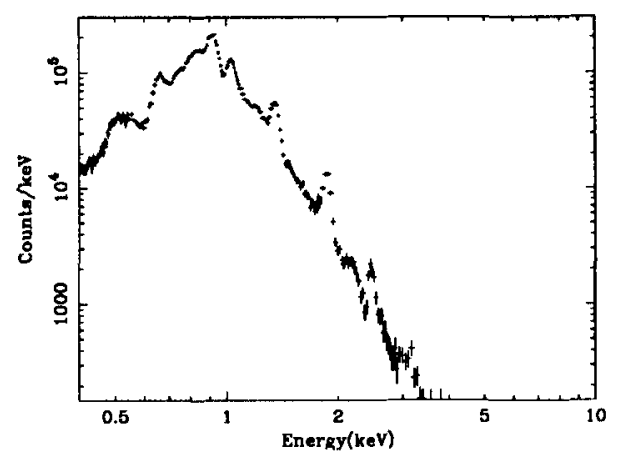

Figure 3. The X-ray spectrum of the eastern knot of Puppis-A.

and the XRT thermal shield. The $S i$ and $O$ edges come from the dead layer of the CCD that is made of $\mathrm{SiO}_{2}$ and $\mathrm{Si}$.

\subsection{Puppis- $A$}

Puppis-A has an incomplete shell-structure with a radius of about 30 arcmin (Gorenstein et al., 1974, Levine et al., 1979). Its X-ray emission comes from a thin thermal plasma that contains many emission lines (Winkler et al., 1981). There are several bright knots showing the inhomogeneities in the interstellar matter (Petre et al., 1982).

Fig. 3 shows the X-ray spectrum of the eastern bright knot. There are several emission lines clearly resolved. Most of these are $\mathrm{K}-\alpha$ emission lines from $O, N e, M g, S i$ and $S$. The complicated structure around $0.7 \sim 0.9 \mathrm{keV}$ mainly comes from $\mathrm{Fe}-L$ emissions. The emission above $5 \mathrm{keV}$ is just below the detection limit of $A S C A$. The continuum spectrum shows the temperature to be about $0.3 \mathrm{keV}$. During the PV phase, we planned to observe the whole remnant with the SIS.

\section{3. $W 49 B$}

W49B has a shell structure in the radio band while it shows a center-filled structure in Xray (Pye et al., 1984). It is classified as a plerion-type SNR in the X-ray region (Seward, 1990). Smith et al. (1985) reported on the X-ray spectrum up to $10 \mathrm{keV}$ with EXOSAT and obtained an $\mathrm{Fe}-K$ emission line with an equivalent width of about $4.7(+0.2-0.6)$ $\mathrm{keV}$. Other line features have not been clearly resolved due to the energy resolution of the EXOSAT detector.

Fig. 4 shows the X-ray spectrum obtained with the $S I S$. There are several emission lines easily resolved. Most of them are $\mathrm{K}-\alpha$ line emissions from helium-like and hydrogenlike ions of $S i, S, A r, C a$ and $F e$. The continuum spectrum can be well fitted with a thermal Bremsstrahlung model of about $2 \mathrm{keV}$. However, the emission line ratios between the helium-like ion and the hydrogen-like ion show that these elements have not reached collisional ionization equilibrium at this temperature. We found that the spectrum could not be reproduced with a simple non-equilibrium ionization (NEI) model which is parameterized with a single electron temperature and a single plasma parameter (the product of the electron density and the elapsed time after the shock heating).

The X-ray spectrum shows a strong cutoff at around $1 \mathrm{keV}$. We found that the column density is about $3 \times 10^{22} \mathrm{H}$ atom $\mathrm{cm}^{-2}$, which is consistent with that obtained by Smith et al. (1985). W49B is expected to be a typical shell-like SNR taking into account 


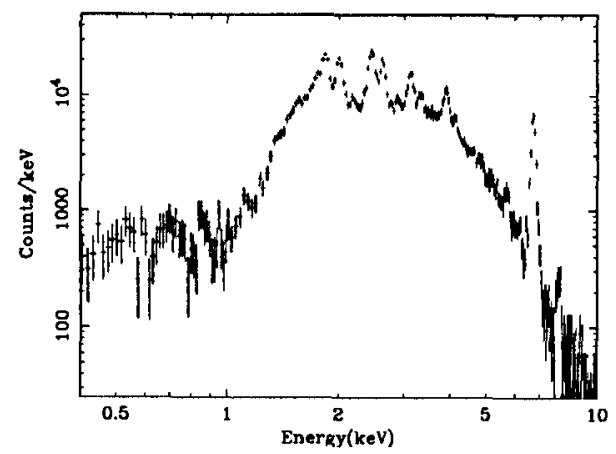

Figure 4. The X-ray spectrum of W49B.
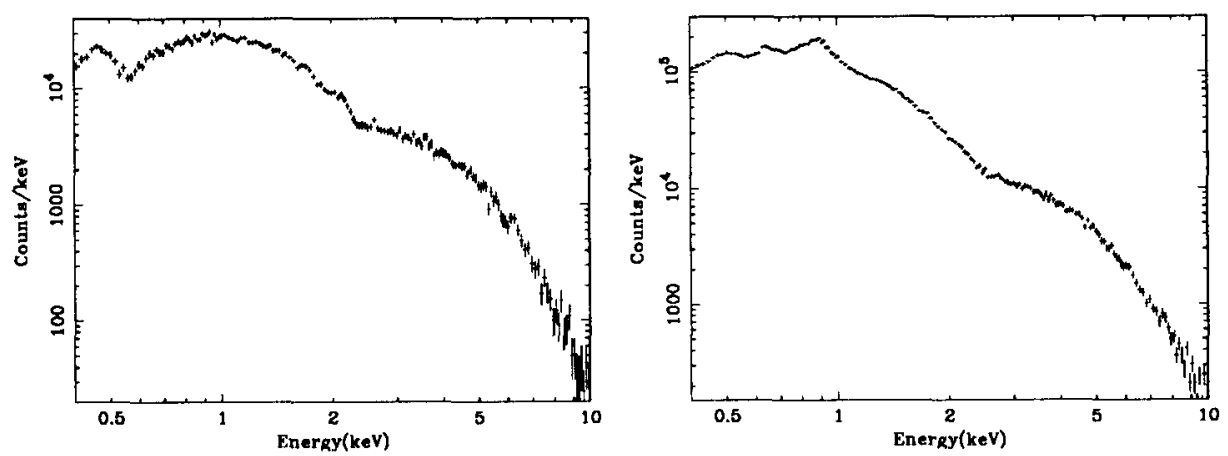

FIgURE 5. The X-ray spectrum of Vela-X (Vela pulsar) (5a, left) and its surrounding nebula $(5 \mathrm{~b}$, right $)$.

the facts that there is no clear point-like source inside the remnant and that the X-ray emission is thermal in origin. The emission can be roughly divided into two parts: one is from the reverse shock and the other is from the forward shock. The emission from the reverse shock shows a higher temperature than that from the forward shock. We can understand that the low-temperature emission from the forward shock is absorbed while the high-temperature emission from the reverse shock is seen.

W49B is about $2 \sim 3$ arcmin in angular diameter. Since the PSF of the XRT is a little smaller than this size, we may be able to study the spectral distribution of the $\mathrm{X}$-ray image. According to preliminary analysis, the extent of each X-ray emission line is slightly different for each ion. The higher $\mathrm{Z}$ elements show a smaller size. This result suggests an onion-skin structure in the reverse shock. A detailed analysis will be done elsewhere.

\subsection{Vela-X}

Fig. 5a,b show the X-ray spectra of Vela-X and its surrounding area obtained with the SIS. The spectrum for Vela-X is obtained from a circular area with a radius of 1.5 arcmin 


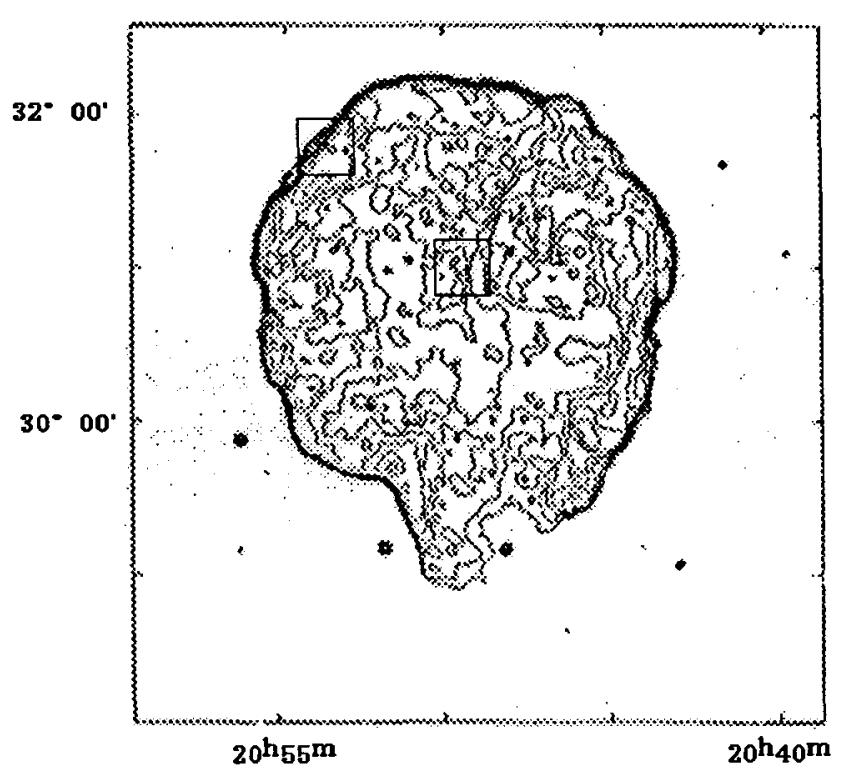

Figure 6. The whole image of the Cygnus Loop observed with Einstein (Seward, 1990). Rectangles show the NE rim and the central region which were observed with the SIS.

centered on the Vela pulsar. The spectrum of the surrounding area is obtained from the region that is more than 5 arcmin away from the Vela pulsar. The spectrum of Vela pulsar is similar to that of Crab. There are no clear emission lines. Some instrumental spectral features due to the XRT and the SIS are evident. In contrast, the spectrum of the surrounding region shows some emission line features, probably due to the $O-K$ line and $F e-L$ line blends. We should note that the spectrum of fig. $5 \mathrm{~b}$ is partly contaminated by the Vela pulsar due to the PSF of the XRT.

\subsection{Cygnus Loop}

The Cygnus Loop is one of the strongest X-ray sources below $1 \mathrm{keV}$. It exhibits a typical shell-structure about 3 degrees in diameter. Ku et al. (1984) measured the temperature of the shell region to be about $2 \times 10^{6} \mathrm{~K}$ and found a temperature gradient inside the shell. Tsunemi et al. (1988) observed emission lines of $S i$ and $S$ from the whole remnant with GSPC on Tenma. Hatsukade and Tsunemi (1990) reported on emission above 1.5 $\mathrm{keV}$ and found its extent to be smaller than that below $1.5 \mathrm{keV}$. Therefore, we can expect that the emission from the shell region shows a low temperature with a high emission measure while regions inside the shell show a high temperature with a low emission measure. Vedder et al. (1986) observed a small portion of the northern rim with the FPCS on Einstein. They showed that the plasma did not reach the collisional ionization condition based on observations of the oxygen lines.

We observed two small regions of the Loop shown in fig. 6. The rectangles in this figure show the field of view of the SIS. One pointing was centered on the north-eastern bright (NE) rim and the other pointing was centered on the central region. The $\mathrm{X}$-ray image for the NE rim is shown in fig. 7.

The X-ray spectra for these two regions are shown in figs. 8a,b. The X-ray spectrum of the central region shows emission lines of $S$ and $S i$. The broad hump around $0.8 \mathrm{keV}$ mainly comes from $\mathrm{Fe}-L$ line blends. The NE rim spectrum is different than the central region spectrum. There is a clear emission line from $M g$ while there are almost no lines 


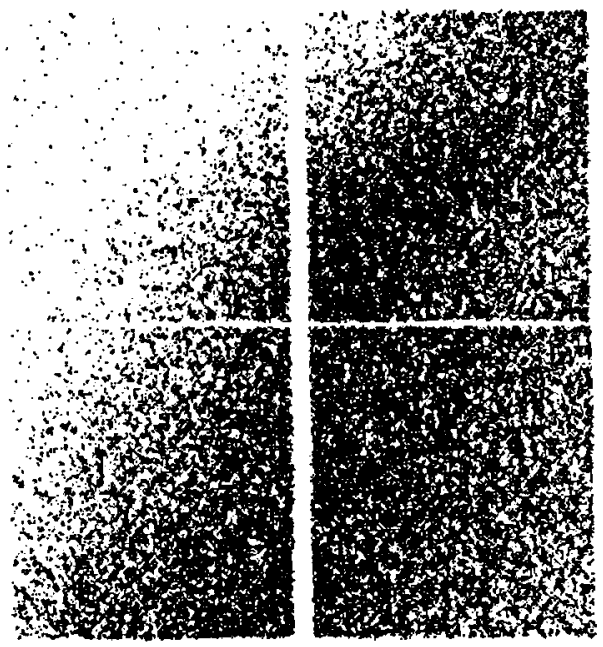

Figure 7. The X-ray image of the NE rim of the Cygnus Loop shown in the left figure obtained with the SIS (four CCD chips). The gap between the CCD chips is seen.
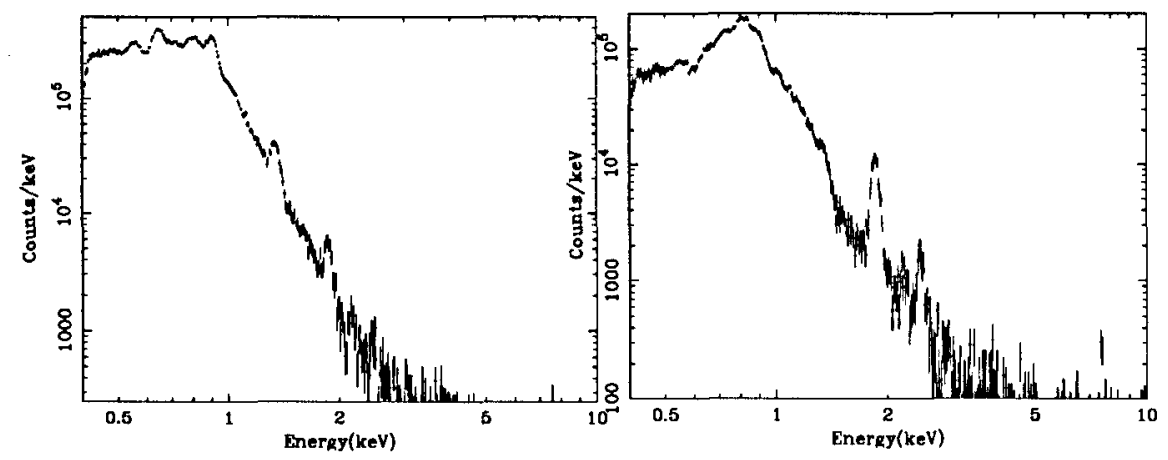

Figure 8. X-ray spectra of two parts of the Cygnus Loop (see Fig. 6). Fig. 8a (left) - north east (NE) rim; Fig. 8b (right) - central region.

from $S$. Furthermore, there are clear emission lines of $O V I I, O V I I I$ and $N e I X$. These differences show that the temperature in the central region is much higher than that in the NE region.

We found that a single temperature collisional ionization equilibrium model does not fit the X-ray spectrum of the NE region. The average plasma parameter in the NE rim is about $10^{11 \sim 12} \mathrm{~cm}^{-3} \mathrm{~s}$ with an electron temperature $0.2 \sim 0.25 \mathrm{keV}$. The spatially resolved analyses along the radial direction of the shell will reveal the plasma structure of the shock front. 


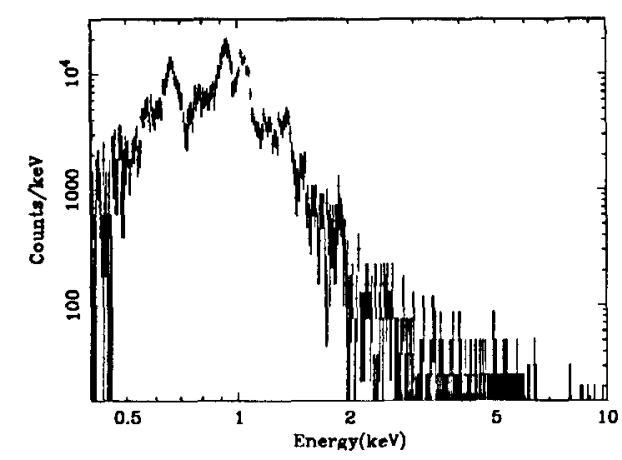

Figure 9. The X-ray spectrum of E0102-72

\section{6. $E 0102-72$}

E0102-72 is the second brightest $\mathrm{X}$-ray source in the SMC at X-ray energies. It was discovered in the Einstein IPC survey of the SMC (Seward and Mitchell, 1981). The observation with $H R I$ on Einstein (Inoue et al., 1983) revealed a shell structure with a diameter about 30 arcsec. The optical observation (Dopita et al., 1981) shows that it is a young, oxygen-rich SNR.

Fig. 9 shows the X-ray spectrum obtained with the SIS. We can see the K- $\alpha$ emission lines from $N e-I X$ and $N e-X$ as well $O-V I I I$. The emission line from $O-V I I$ seems to be quite strong since it is seen in the trough due to the oxygen absorption edge in the CCD response. The intensity of $F e-L$ line blends is quite weak compared to those of $\mathrm{Ne}$ and $\mathrm{O}$. These results are consistent with those discussed by Hughes (1987). The spatial extent of this source is small, such that it looks like a point source with $A S C A$. Therefore, we can get only the overall spectrum. 




Figure 10. The X-ray spectrum of Cassiopeia-A.

FIGURE 11. The X-ray image of Cassiopeia-A obtained with the SIS (one CCD chip). North is up. A scar from the center to the upper right is due to a bad column in the CCD.

\subsection{Cassiopeia- $A$}

Cas- A forms a circular structure both in X-rays and in radio with a diameter of $3 \sim 4$ arcmin. Becker et al. (1979) discovered many emission lines with SSS on Einstein. It is believed to be the result of a type II SN. Tsunemi et al. (1986) observed Cas-A with $G S P C$ on Tenma and found that the plasma did not reach collisional ionization equilibrium. They obtained the mean energy of the $F e-K$ line blends to be about 6.6 $\mathrm{keV}$.

Fig. 10 shows the X-ray spectrum obtained with the SIS. There are several emission lines. In particular, the $F e-K$ line blend seems to be broader than that of the detector response. Markert et al. (1983) obtained the Doppler shift broadening of $5000 \mathrm{~km} / \mathrm{sec}$ by using the FPCS on Einstein. The energy resolution of the SIS should be able to see this broadening. Fig. 11 shows the $\mathrm{X}$-ray image with the SIS. The image quality is restricted by the PSF of the XRT. We noticed that there are two bright parts. We will be able to get some spatially resolved spectra. 


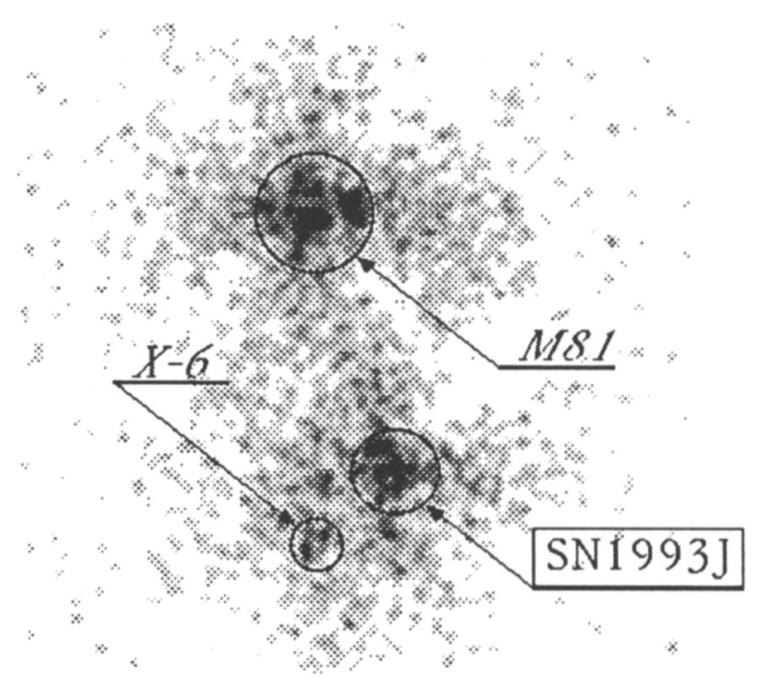

FIgURE 12. The X-ray image around SN1993J observed with the SIS. There are three sources seen here. Three statistically significant sources are seen. North is up. See text.

\subsection{SN1993J}

When a SN appeared in M81 at the end of March, 1993 (Ripero, 1993), we rescheduled the observation plan and observed the M81 region on April 7. We detected X-rays from SN1993J in the energy range of 0.4 to $12 \mathrm{keV}$ (Tanaka et al., 1993). Fig. 12 shows the $\mathrm{X}$-ray image obtained with the SIS. The complicated intensity structure is due to the Poisson statistics as well as the pattern of the PSF of the XRT. There are three peaks in the figure which are shown by circles. The strongest one in the north comes from X-5 of M81 (the core of M81). The second strongest one in the lower east coincides with the location of SN1993J. About 1 arcmin to the west of SN1993J, there is the known X-ray source X-6 in M81. We continued observations until mid-May when we met a solar angle constraint on the satellite. The $\mathrm{X}$-ray intensity monotonically decayed with an e-folding time about $50 \sim 70$ days. The maximum luminosity was about $5 \times 10^{39} \mathrm{erg} \mathrm{s}^{-1}$. We plan to observe SN1993J again when the solar angle constraint allows.

\section{Conclusion}

We report here initial results of SNR observations with $A S C A$ 's SIS detectors. The energy resolving power of the SIS is much better than the non-dispersive instruments employed in previous satellites. It can easily separate the $\mathrm{K}-\alpha$ emission of helium-like ions from that of hydrogen-like ions. We have learned that the most of the thin hot plasma in SNRs observed thus far have not reached the collisional ionization equilibrium condition. $A S C A$ will reveal the plasma condition in detail by resolving many emission lines. In addition, the ability to make spatially resolved spectra enables us to study the evolution of hot plasmas. 


\section{Acknowledgments}

The author expresses his special thanks to all the members of the $A S C A$ team. E. Miyata helped me to arrange the Tex format. Dr. K. C. Gendreau read the manuscript carefully.

\section{REFERENCES}

Becker, R. H., Boldt, E. A., Holt, S. S., Mushotzky, R. F., Serlemitsos, P. J., Smith, G. W., and White, N. E. (1979), ApJ, 234, L73.

Burke, B. E., Mountain, R. W., Harrison, D. C., Bautz, M. W., Doty, J. P., Ricker, G. R., and Daniels, R. J., (1991), IEEE Trans. ED-38, 1069.

Burke, B. E., Mountain, R. W., Daniels, R. J., and Cooper, M. J. (1993), SPIE, 2006-31.

Dopita, M. A., Tuohy, I. R., and Mathewson, D. S. (1981), ApJ, 248, L105.

Gorenstein P., Harnden, F. R. Jr., and Tucker, W. H. (1974), ApJ, 192, 661.

Hatsukade, I. and Tsunemi, H. (1990), ApJ, 362, 566

Hughes, J. P., (1987), in IAU Colloq. 101, Supernova Remnants and the Interstellar Medium, ed. R. S. Roger and T. L. Landecker (Cambridge University Press), p. 125)

Inoue, H., Koyama, K., and Tanaka, Y. 1983, in IAU Symp. 101, Supernova Remnants and Their X-ray Emission, ed. I. J. Danziger and P. Gorenstein (Dordrecht: Reidel), p. 535)

Ku, W. H.-M, Kahn, S. M., Long, L, S., and Pisarski, R. L., (1984), ApJ, 278, 615.

Markert, T. H., Canizares, S. R., Clark, G. W., and Winkler, P. F., Jr. (1983), ApJ, 268, 134.

Ohashi, T., Makishima, K., Ishida, M., Tsuru, T., Tashiro, M., Mihara, T., Kohmura, Y., and Inoue, H. (1991), SPIE, 9-19, 1549.

Peter, R., Canizares, C. R., Kriss, G. A., and Winkler, P. F., Jr. (1982), ApJ, 258, 82.

Pye, J. P., Becker, R. H., Seward, F. D., and Thomas, N. (1984), MNRAS, 207, 649.

Ripero, J., (1993), IAUC, No.5731.

Seward, F. D. and Mitchell, M., (1981), ApJ, 243, 736.

Seward F. D., (1990), ApJS, 73, 781.

Smith, A., Jones, L. R., Peacock, A., and Pye, J. P. (1985), ApJ, 296, 469.

Tanaka, Y., and ASCA team. (1993), IAUC, No.5753.

Tsunemi, H., Yamashita, K., Masai, K., Hayakawa, S., and Koyama, K. (1986), ApJ, 306, 248.

Tsunemi, H., Manabe, M., Yamashita, K., and Koyama, K. (1988), Pub. Astr. Soc. Japan, 40, 449 .

Vedder P. W., Canizares, C. R., and Markert, T. H. (1986), ApJ, 307, 269.

Winkler P. F., Jr., Canizares, C. R., Clark, G. W., Kalata, K., Markert, T. H., and Schnopper, H. W., (1981), ApJ, 246, L27. 